\title{
28 Research Square \\ Comparison on FTIR Spectrum and Thermal Analysis for Four Types of Rehamnnia Glutinosa and Their Extracts
}

\author{
Maosen Zhang \\ Nanjing University of Chinese Medicine \\ Rong Ding ( $\nabla$ 865823139@qq.com) \\ Nanjing University of Chinese Medicine \\ Fang $\mathrm{Hu}$ \\ Suzhou Traditional Chinese Medicine Hospital
}

\section{Research Article}

Keywords: Rehmannia glutinosa, extracts, FTIR spectroscopy, thermal analysis

Posted Date: February 17th, 2022

DOl: https://doi.org/10.21203/rs.3.rs-1319441/v1

License: (c) (i) This work is licensed under a Creative Commons Attribution 4.0 International License. Read Full License 


\section{Abstract}

Rehmannia glutinosa ( $R$. glutinosa) is a plant material and widely used clinically in China. Due to different processing methods and various healing effects, there are four types of $R$. glutinosas (RR, RRP, RRC, and RRPC) and their corresponding extracts for convenient use. As complicated mixture systems, chemical components of $R$. glutinosa are very difficult to identify and discriminate. In order to effectively control its quality during processing, Fourier transform infrared spectroscopy (FTIR), second derivative spectrum, and thermal analysis were used in this study. It was found that the major active ingredients were retained in the extracts when extracted from their corresponded $R$. glutinosa and subtle differences could be distinguished by the second derivative spectrum. FTIR spectrum, second derivative spectrum, and thermal analysis are valid method to validate and identify plant material, traditional Chinese medicine material, as well as their corresponding extracts.

\section{Introduction}

Rehmannia glutinosa (also known as $R$. glutinosa) is an edible plant material and a traditional Chinese medicine with extraordinary pharmaceutical value and a long history of medicinal use in China. Studies have shown that the main active ingredients of $R$. glutinosa are polysaccharides and catalpol. $R$. glutinosa polysaccharides can boost immunity, act as antitumor agents, regulate blood lipid concentrations, and replenish blood. Additionally, $R$. glutinosa polysaccharides have hypoglycemic effects, reduce triglyceride and cholesterol levels in animal models, and considerably reduce glucose metabolism abnormalities in rats with chronic stress on a high-fat diet. $R$. glutinosa polysaccharides can also promote the formation of serum circulating hemolysin and hemolytic antibody plaques in normal and CTX-induced immunosuppressed mice. $R$. glutinosa polysaccharides induce anti-anxiety effects by enhancing serum lysozyme activity ${ }^{1-8}$ Also, $R$. glutinosa polysaccharides have substantial effects on diabetic nephropathy ${ }^{9}$, and they can improve cholinergic function and reduce D-galactose-induced inflammatory cytokines in aging mice ${ }^{10}$. Numerous Chinese researchers have isolated polysaccharides from $R$. glutinosa and conducted extensive studies on its chemical structures and pharmacologic actions ${ }^{11-22}$. The nature and efficacy of $R$. glutinosa change after it is processed. Typically, there are four types of preparations for $R$. glutinosa, which are Radix Rehmanniae (RR), Radix Rehmanniae Praeparata (RRP), Radix Rehmanniae Charcoal (RRC), and Radix Rehmanniae Charcoal Praeparata (RRPC) according to application of traditional Chinese medicine. RR is obtained by processing and roasting of fresh $R$. glutinosa and its nature is cold. RRP is obtained from RR through steaming and drying, and its nature is warm ${ }^{1,2}$. RRC and RRPC are obtained from RR and RRP, respectively, by the same processing, their surfaces are partially carbonized. RRC has the effect of cooling blood and stopping bleeding. RRPC is mainly used on blood-suppressing and hemostasis, and is often used to treat uterine bleeding or debilitating bleeding ${ }^{10}$. Therefore, different processed products of $R$. glutinosa have different medicinal uses. Liu's research showed that the contents of catalpol in different $R$. glutinosa products were listed as the following order: RR $>$ RRP $>$ RRC $>$ RRCP, indicating that the processed method has an important influence on $R$. glutinosa ${ }^{11}$.

Traditional Chinese medicine extract has the advantages of free cooking, direct use, safe and hygienic, easy to carry and preserve, and etc. The four types of R. glutinosa are often processed into their extracts (RRE, 
RRPE, RRCE, and RRPCE). Nowadays, there are considerable study reports on pharmacological effects of $R$.

glutinosa $^{15-23}$. However, there were relatively less researches on the similarities and differences of RR, RRP, RRC, RRPC and their extracts (RRE, RRPE, RRCE, RRPCE) by means of FTIR spectroscopy and thermal analysis. FTIR spectroscopy has fingerprint characteristics with strong features; it is the main method for drug identification in various pharmacopoeias ${ }^{24}$. Thermal analysis is also a primary method for quality control of drugs according to its record in pharmacopoeia ${ }^{24-28}$. In this study, the similarities and differences of four types of $R$. glutinosa and their extracts were investigated by FTIR spectroscopy, second derivative spectrum and thermal analysis. The aim of this study is to develop an effective analysis method for studying integrally the main constituents in the complicated mixture systems such as plant materials and their corresponding extracts.

\section{Experiments}

\subsection{Materials}

Four types of R. glutinosa (RR, RRP, RRC, and RRPC) were obtained from Jiaozuo, Henan Province, China. Four types of R. glutinosa extracts (RRE, RRPE, RRCE, and RRPCE) were purchased from Jiangsu Jiangyin Tianjiang Pharmaceutical Co. Ltd (Jiangyin, China). Their pictures were as follows:

\subsection{Methods}

\subsubsection{FTIR spectral analysis}

The sample of four types of R. glutinosa and their extracts were vacuum dried $\left(110^{\circ} \mathrm{C}, 2 \mathrm{~h}\right)$, ground into powder, and sieved with a 80 -mesh screen. The powder $(0.002 \mathrm{~g})$ was thoroughly mixed with the $\mathrm{KBr}$ powder $(0.2 \mathrm{~g})$ and pressed into a pellet for measurement by a Fourier transform infrared (FTIR) spectrophotometer (Nicolet iS-1063001, Thermo Fisher Scientific (China) Co. Ltd.) in the range of $400-4000 \mathrm{~cm}^{-1}$ with a resolution of $4 \mathrm{~cm}^{-1}$. An average of 16 scans was used for each sample. In this study, the second derivative spectra of FTIR spectrograms were analyzed by Omnic 8.3 software and SPSS 22.0 software.

\subsubsection{Similarity:}

Correlation coefficient is a common method evaluating the similarity for Infrared spectroscopy and other fingerprints of traditional Chinese medicine. In this study, the correlation coefficient was obtained using the calculation function of origin software, which indicates the similarity of two infrared spectra matching each other. The similarity was evaluated by the correlation coefficient of four types of R. glutinosa and their extracts. The correlation coefficient formula is as follows:

\subsubsection{Precision experiment}

A sample of RR was taken for infrared spectral analysis by measuring five times continuously. The similarity value (correlation coefficient) was calculated by SPSS 22.0. The similarity values were 0.999970, 0.999997, 0.999997, 0.999997, and 0.999951, respectively. All data were shown as relative standard deviation $(\mathrm{RSD}=0.02 \%$ o), respectively. The high similarity demonstrated the instrument with a high precision. 


$$
p=\frac{\sum_{i=1}^{n}\left(x_{i}-\bar{x}\right)\left(y_{i}-\bar{y}\right)}{\sqrt{\sum_{i=1}^{n}\left(x_{i}-\bar{x}\right)} \sqrt{\sum_{i=1}^{n}\left(y_{i}-\bar{y}\right)^{2}}}
$$

\subsubsection{Stability experiment}

A sample of RR was taken for infrared spectral analysis by measuring five times every hour. The similarity value (correlation coefficient) was calculated by SPSS 22.0 . The similarity values were $0.999993,0.999996$, $0.999994,0.999994$, and 0.999977 , respectively. All data were shown as relative standard deviation $(\mathrm{RSD}=0.008 \%$ ). The high similarity indicated that the samples had a high stability.

\subsubsection{Reproducibility experiment}

Five samples of RR were prepared in parallel, and the infrared spectra of the samples were determined, respectively. The similarity value (correlation coefficient) was calculated by SPSS 22.0. The similarity values were $0.987412,0.986810,0.999997,0.994340,0.994825$, and 0.9985 , respectively. All data were shown as relative standard deviation $(\mathrm{RSD}=0.55 \%)$. The high similarity indicated that the proposed method had a high reproducibility.

\subsubsection{Thermal analysis}

Thermal analysis experiments of the samples of four types of R. glutinosa and their extracts were carried out under nitrogen atmosphere in a thermal analyzer (NETZSCH STA449 F3 Jupiter, Hexico Scientific Instruments (Shanghai) Co., Ltd.). The TG (Thermal Gravity Analysis) curves were conducted at heating rates in the range of $20 \mathrm{C} / \mathrm{min}$ from room temperature to $800^{\circ} \mathrm{C}$ in a nitrogen atmosphere $(20 \mathrm{ml} / \mathrm{min})$. The sample amount used was between 10 and $15 \mathrm{mg}$ per specimen, and the collected data were used for further analysis.

\section{Results And Discussion}

\subsection{FTIR spectra analysis of four types of $R$. glutinosa and their extracts}

The chemical components of $R$. glutinosa mainly include polysaccharides, oligosaccharides, iridoid glycosides (catalpol was the main element of iridoid glycosides in $R$. glutinosa), amino acids, and etc. These were material basis for the pharmacological effects of $R$. glutinosa. Fig. 2 illustrated the FTIR spectra of four types of $R$. glutinosa and their extracts. Detailed peak positions and assignments of the samples were summarized in Tab. 1. As shown in Fig. 2 and tab 1, FTIR spectra of four types of $R$. glutinosa and their extracts showed higher similarities and typical absorption peaks of polysaccharides, oligosaccharides, catalpol, amino acids, and etc. There were 7 groups of typical common peaks. These absorption peaks were as follows. 1) -OH stretching vibration of the hydroxide radical from $R$. glutinosa polysaccharides, oligosaccharides, and catalpol molecules was near $3418 \mathrm{~cm}^{-1}$. 2) $-\mathrm{CH}$ stretching vibration of methyl and methylene from polysaccharides and oligosaccharides was near $2926 \mathrm{~cm}^{-1}$. 3) $\mathrm{C}=0$ stretching vibration of polysaccharides and amide I band produced by bending vibration of - $\mathrm{NH}$ group in amino acid molecules was 
near $1636 \mathrm{~cm}^{-1} .4$ ) the peak of $1419 \mathrm{~cm}^{-1}$ was attributed by the variable angle vibration of $\mathrm{C}-\mathrm{H}$ from polysaccharides and oligosaccharides. 5) C-O stretching vibration of glycoside molecules was near 1263 $\left.\mathrm{cm}^{-1} .6\right)$ the region of $1145-1025 \mathrm{~cm}^{-1}$ corresponded to ring vibrations overlapped with stretching vibrations of $(\mathrm{C}-\mathrm{OH})$ side groups and $(\mathrm{C}-\mathrm{O}-\mathrm{C})$ glycosidic bend vibration of polysaccharides and oligosaccharides. 7) C-O stretching vibration of carbohydrates and glycosides was near $586 \mathrm{~cm}^{-1}$. In a nutshell, there were many common features in FTIR spectra of four types of $R$. glutinosa and their extracts. These indicated that the chemical components of four types of $R$. glutinosa and their extracts were basically the same.

Table 1

Peak positions of FTIR of four types of $R$. glutinosa and their extracts

\begin{tabular}{|lllllllllllllll|}
\hline sample & \multicolumn{1}{l}{ Wave number $/ \mathrm{cm}^{-1}$} \\
\hline RR & 3418 & 2926 & 1636 & 1518 & 1419 & 1263 & 1145 & 1070 & - & - & - & 598 \\
RRP & 3421 & 2929 & 1636 & - & 1419 & 1260 & 1145 & 1075 & - & 871 & 799 & 586 \\
\hline RRC & 3431 & 2926 & 1636 & - & 1419 & 1238 & 1149 & 1072 & - & - & - & 529 \\
\hline RRPC & 3426 & 2924 & 1636 & - & 1419 & 1259 & 1150 & 1075 & - & - & - & 586 \\
RRE & 3407 & 2926 & 1617 & - & 1419 & 1230 & 1150 & 1075 & 1026 & 899 & 772 & 578 \\
RRPE & 3384 & 2924 & 1636 & - & 1419 & 1239 & 1151 & 1077 & 1025 & 867 & 774 & 578 \\
\hline PPCE & 3407 & 2926 & 1635 & - & 1418 & 1240 & 1150 & 1078 & 1025 & 868 & 770 & 578 \\
\hline RRPCE & 3387 & 2927 & 1635 & & 1413 & 1237 & 1151 & 1078 & 1025 & 867 & 766 & 578 \\
\hline
\end{tabular}

As shown in Fig. 2a, the intensities of absorption peaks of RRP were stronger than those of RR at around $3418 \mathrm{~cm}^{-1}$ and $2926 \mathrm{~cm}^{-1}$, and the absorption peaks were observed at $871 \mathrm{~cm}^{-1}$ and $799 \mathrm{~cm}^{-1}$ for RRP. This was due to RRP was obtained from RR through processing, steaming, and drying. When RR was processed and processed into RRP, polysaccharides and oligosaccharides were converted to monosaccharides (galactose, fructose, and glucose). According to the literature, monosaccharides made RRP "sweet as sugar", fructose could react with amino acids to form melanin, which made RRP "black as lacquer", and the monosaccharide content of RRP was more than twice of RR ${ }^{1,22}$, which was the reason of RRP to turn black. Due to the presence of $-\mathrm{OH}$ and $-\mathrm{CH}$ in the galactose, fructose, and glucose molecules, the intensities of absorption peaks in $3418 \mathrm{~cm}^{-1}$ and $2926 \mathrm{~cm}^{-1}$ of RRP were stronger than those of RR. It could also be seen from Fig. 2a that the intensities of absorption peaks near $3418 \mathrm{~cm}^{-1}, 2926 \mathrm{~cm}^{-1}, 1636 \mathrm{~cm}^{-1}$, and $1263 \mathrm{~cm}^{-1}$ were significantly reduced in RRC and RRPC, which indicated lower levels of polysaccharides,

oligosaccharides, catalpol, amino acids, and etc. The reason was that RRC and RRPC were obtained from RR and RRP, respectively. Partial carbonization occurred during processing, which reduced the intensities of the characteristic peaks of polysaccharides, oligosaccharides, and catalpol hydroxyl groups. The order of the intensities of absorption peaks of four types of $R$. glutinosa were RRP> RR> RRPC> RRC near $3418 \mathrm{~cm}^{-1} \mathrm{and}^{-}$ $2926 \mathrm{~cm}^{-1}$.

As shown in Fig. 2b, the intensities of absorption peaks of RRPE were slightly stronger than those of RRE at around $3407 \mathrm{~cm}^{-1}$ and $2926 \mathrm{~cm}^{-1}$, while RRCE and RRPCE were slightly lower. The peak intensities at around 
$3418 \mathrm{~cm}^{-1}$ and $2926 \mathrm{~cm}^{-1}$ of four types of extracts exhibited the same order as corresponding R. glutinosa, which were RRPE> RRE > RRPCE > RRCE. Therefore, it could be concluded that RRE and RR, RRPE and RRP, RRCE and RRC, and RRPCE and RRPC share similar chemical components, respectively. The absorption peaks were observed at around $1025 \mathrm{~cm}^{-1}, 867 \mathrm{~cm}^{-1}$, and $774 \mathrm{~cm}^{-1}$ for four types of $R$. glutinosa extracts while no obvious corresponding peaks were seen for four types of $R$. glutinosa except RRP at $871 \mathrm{~cm}^{-1}$ and $799 \mathrm{~cm}^{-1}$. The reason might be that four types of extracts were extracted from corresponding $R$. glutinosa respectively, and a small amount of malt dextrin were added during extraction, which could cause absorption peaks at around $1025 \mathrm{~cm}^{-1}, 867 \mathrm{~cm}^{-1}$, and $774 \mathrm{~cm}^{-1}$. It could also be seen from Tab. 1 that the absorption peaks moved towards low wavenumber near $3418 \mathrm{~cm}^{-1}$ and $1260 \mathrm{~cm}^{-1}$ for four types of $R$. glutinosa extracts, and these might be caused by malt dextrin. In addition, the absorption peaks disappeared at $1518 \mathrm{~cm}^{-1}$, which mainly corresponded to amide I band and amide II in RRE. Thus, it could be deduced that the relative content of protein and plant acid in RRE were lower than those in RR. The characteristic absorption peaks of excipient malt dextrin were at around $1025 \mathrm{~cm}^{-1}, 867 \mathrm{~cm}^{-1}$, and $774 \mathrm{~cm}^{-1}$.

\subsection{Comparative analysis of FTIR spectra of four types of $R$. glutinosa and their extracts}

Figure 3 presented a comparison of FTIR spectra and relative intensity of absorption peaks of extracts with those of corresponding $R$. glutinosa (relative intensity of absorption peaks was the ratio of the intensities of 5 groups of characteristic peaks to those of the maximum peak at around $3400 \mathrm{~cm}^{-1}$ respectively). As shown in Figure 3, the main absorption peak positions and shapes of four types of extracts were quite similar with those of corresponding $R$. glutinosa. Comparison between two spectra in Figure 3a-3d revealed that the main specific peak positions and shapes were fairly similar to each other for RR and RRE, as well as for RRP and RRPE, RRC and RRCE, and RRPC and RRPCE, respectively, which demonstrated that four types of $R$. glutinosa extracts and corresponding $R$. glutinosa had similar chemical compositions. However, the differences were also observed that some absorption peak intensities and absorption peak width of the extracts were wider than those of $R$ glutinosa at around $3418 \mathrm{~cm}^{-1}$. The reason was caused by adding malt dextrin.

It could also be seen from Fig. 3 that the relative intensity of peaks showed differences at around $1636 \mathrm{~cm}^{-1}$, $1419 \mathrm{~cm}^{-1}, 1240 \mathrm{~cm}^{-1}, 1150 \mathrm{~cm}^{-1}$ and $1075 \mathrm{~cm}^{-1}$ for four types of R. glutinosa and their extracts (i.e. RR and RRE, RRP and RRPE, RRC and RRCE, RRPC and RRPCE ). For example, the relative intensity of peak near $1636 \mathrm{~cm}^{-1}$ of RR was higher than that of RRE, and slight differences near $1419 \mathrm{~cm}^{-1}$ and $1240 \mathrm{~cm}^{-1}$, whereas the relative intensity of peaks near $1150 \mathrm{~cm}^{-1}$, and $1075 \mathrm{~cm}^{-1}$ of RR were lower than those of RRE. The peak of $1636 \mathrm{~cm}^{-1}$ was produced by $\mathrm{C}=0$ stretching vibration of polysaccharides and amide I band by bending vibration of $-\mathrm{NH}$ group in amino acid molecules, It showed that when RR was extracted into RRE, the content of polysaccharides and the amino acids were reduced. The peak of $1419 \mathrm{~cm}^{-1}$ was attributed by the variable angle vibration of $\mathrm{C}-\mathrm{H}$ from polysaccharides and oligosaccharides and the peak of $1150 \mathrm{~cm}^{-1}$ was mainly generated by polysaccharide with hydroxide radical $(\mathrm{C}-\mathrm{OH})$, the region of $1145-1025 \mathrm{~cm}^{-1}$ corresponded to ring vibrations overlapped with stretching vibrations of $(\mathrm{C}-\mathrm{OH})$ side groups and $(\mathrm{C}-\mathrm{O}-\mathrm{C})$ glycosidic bend vibration of polysaccharides and oligosaccharides and $\mathrm{C}-0$ stretching absorption peaks were identified at 899 $\mathrm{cm}^{-1}$ and $772 \mathrm{~cm}^{-1}$ for glycosides and carbohydrates, respectively. Therefore, glycosides and carbohydrates 
characteristic peaks were more prominent for RRE, and Similarly, RRP and RPE, RRC and RCE, RRPC and RRPCE had the same characteristics as the above analysis. In short, the characteristic peaks of glycosides and carbohydrates were more obvious for four types of extracts.

\subsection{Second derivative spectra of four types of $R$. glutinosa and their extracts}

Generally, the second derivative infrared spectrum could greatly enhance the spectral resolution and amplify tiny differences in FTIR spectrum. Since $R$. glutinosa and their extracts were a mixture of various active components, some absorption peaks were overlapped in FTIR spectra. The second derivative infrared spectroscopy with higher resolution could be applied to further analyze tiny differences of $R$. glutinosa and their extracts. As illustrated in Fig. 4, a number of differences invisible in FTIR spectra became clearer, especially in the range from 1200 to $500 \mathrm{~cm}^{-1}$, which mainly reflected the absorption of $\mathrm{C}-\mathrm{O}$ and $\mathrm{C}-\mathrm{OH}$ vibration of polysaccharides, oligosaccharides, and monosaccharides. There manifested as multiple "sawtooth" peaks in the range of $1200-500 \mathrm{~cm}^{-1}$ wave bands with significant differences in peak number and peak intensities for four types of and their extracts. The peak intensities in the range of $900-770 \mathrm{~cm}^{-1}$ were higher in RRP and RRPE (the absorption peak at $830 \mathrm{~cm}^{-1}$ and $778 \mathrm{~cm}^{-1}$ were caused by a-glycosidic bond). Since RRP was processed by RR, polysaccharides and oligosaccharides of RR were converted into monosaccharides (galactose, fructose, and glucose). RRPE were extracts from RRP. Therefore, RRP and RRPE had greater contents of monosaccharide (fructose and glucose) and their characteristic peaks of monosaccharides were more obvious, by which they could be easily distinguished from others. RRC and RRPC were obtained from RR and RRP, respectively, and with partial carbonization, lower contents of polysaccharides, oligosaccharides, and monosaccharide were in RRC and RRPC, lower numbers of "sawtooth" absorption peaks were in RRC and RRPC, and fewer characteristic peaks of polysaccharides, oligosaccharides, and monosaccharides could distinguish RRC and RRPC with other R. glutinosa and extracts. There were slightly higher contents of glycosides and carbohydrates in four types of extracts due to the addition of malt dextrin excipients during extraction. Thus, slightly higher numbers of "saw-tooth" absorption peaks were in four types of extracts. For example, some peak intensities of RRCE and RRPCE in the range of $1200-700 \mathrm{~cm}^{-1}$ were slightly higher than those of RRC and RRPC. Absorption peaks could also been seen at around $1150 \mathrm{~cm}^{-1}$ and peak intensities were different and conformed to the above analyses in four types of $R$. glutinosa and their extracts. It could be seen that second derivative spectra amplify the differences and reveal the potentially characteristic FTIR absorption bands, as well as enhance the spectral resolution and obtain more new information for distinguishing similar complicated samples. Therefore, it could be concluded that the second derivative infrared spectrum could distinguish four types of $R$. glutinosa and their extracts.

\subsection{The similarity of FTIR of four types of R. glutinosa and their extracts}

The correlation coefficient could indicate the similarity of two infrared spectra matching each other. Tab 2 showed significant similarity of FTIR spectra between the extracts and corresponding $R$. glutinosa, and the similarities were $0.951,0.963,0.960$, and 0.954 for RRE and RR, RRPE and RRP, PPCE and RRC, and RRPCE 
and RRPC, respectively. Among them, RRPE and RRP exhibited the highest similarity (0.963). In addition, the chemical structures of the extracts were similar to those of the corresponding $R$. glutinosa, which further proved that four types of extracts had multiple identical chemical components with their corresponding $R$. glutinosa and retained active ingredients from raw medicinal materials. The minor differences observed between the extracts and corresponding $R$. glutinosa may be caused by small amounts of components that were not completely extracted and the addition of malt dextrin excipients during extraction.

Table 2

The similarity of FTIR of four types of $R$. glutinosa and their extracts

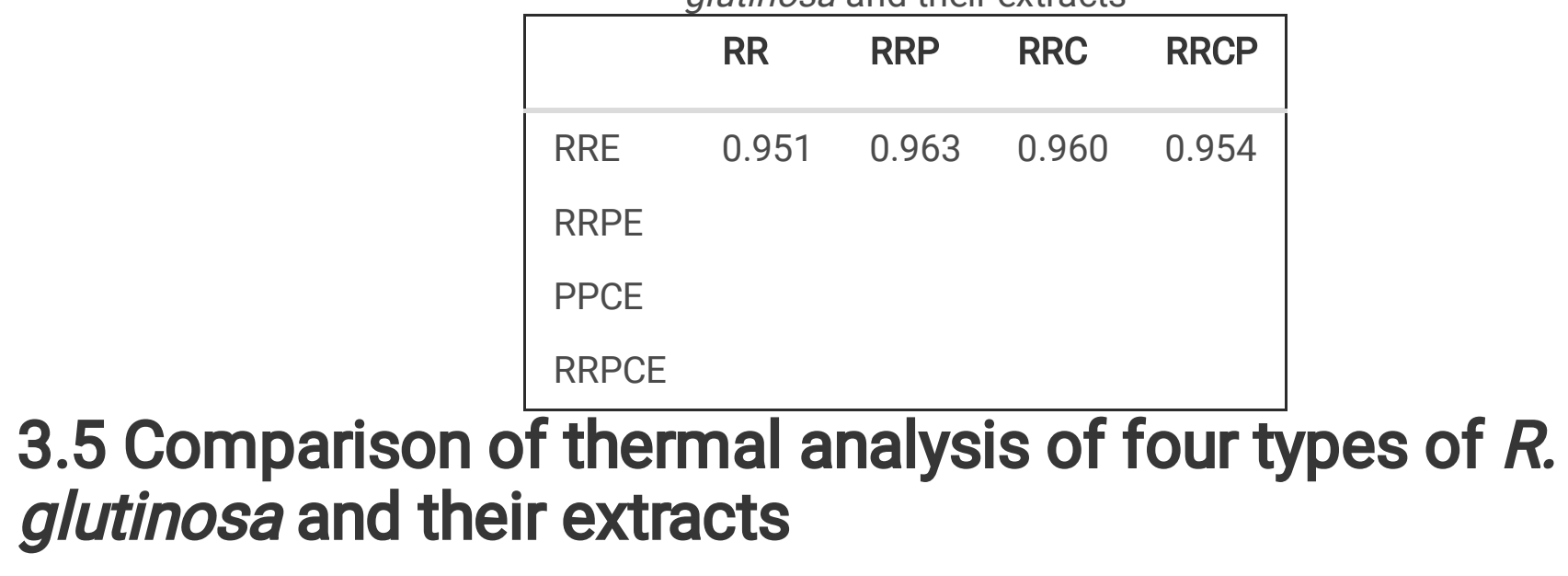

Figure 5 showed a comparison of TG and DTG curves of four types of $R$. glutinosa and their extracts. It could be seen that TG and DTG curves of four types of extracts were very similar to those of corresponding $R$. glutinosa, and the peak positions and shapes of characteristic temperature in DTG curve of four types of extracts were also very similar to those of corresponding $R$. glutinosa, and the same time the TG and DTG curves of RR and RR were similar, while RRC and RRPC were more similar. This proved that four types of $R$. glutinosa extracts retained the effective ingredients of their raw materials. This also proved that the active ingredients of RR and RRP were similar, while PPC and RRPC were more similar, which was consistent with the above spectral analysis. It could be seen from DTG curve in Figure 5 that peak 1 and 3 of RR, RRP and their extracts were not obvious while peak 2 was sharp and larger, indicating a lower rate of mass loss in the first and third stage and a higher rate of mass loss in the second stage (about $\left.190^{\circ} \mathrm{C}-350^{\circ} \mathrm{C}\right)$. The reason could be that RR and its extract were rich in polysaccharides, catalpol and a variety of amino acids, and these organic biomasses, especially polysaccharides would be decomposed into monosaccharides when heated. Monosaccharides would be dehydrated to caramel at $190^{\circ} \mathrm{C}$ to $220^{\circ} \mathrm{C}$. Caramel was subjected to further heating to form carbon dioxide and carbon monoxide at high temperature, resulting in a large mass loss. Thus, RR and its extract had a greater mass loss in the second stage. The same reason was for RRP and its extract. Peak 1 in DTG curves of RRC and RRPC were larger than those of RR and RRP, which indicated that RRC, RRPC had higher mass loss rates in the second stage, at the same time, the mass loss rate of RRC and RRPC were slightly higher than those of RR and RRP in the first stage. The reasons were that RRC and RRPC processed by RR and RRP respectively and they were partially carbonized, and these might caucused them to absorbed water easily, RRC and RRPCs had slightly higher mass loss rate in the first stage $\left(\leq 190^{\circ} \mathrm{C}\right)$ due to the removal of water vapor molecules. RRCE and RRPCE have low water absorption due to processing into extracts. 


\section{Conclusions}

Based on systematical analysis of four types $R$. glutinosa and their extracts using FTIR, second derivative spectrum, and thermal analysis, it can be concluded that these methods could support large numbers of microscope structure information and entire rules of chemical constituents in medicinal materials. By using microscope fingerprint characters of FTIR spectrum, second derivative spectrum, and thermal analysis, the constituents in the extracts can be tested accurately, instantly, and effectively and the quality of medicinal materials can be validated. Thus, FTIR spectrum, second derivative spectrum, and thermal analysis reflecting objectively the panorama of chemical constituents in complex system is the most credible method to validate and identify the mix-substance systems, such as traditional Chinese medicine, herbal medicine, as well as their corresponding extracts.

\section{Declarations}

Funding: This work was supported by the Natural Science Foundation Youth Fund of China (No. 81503299).

Statement: The materials used in this study comply with relevant institutional, national, and international guidelines and legislation.

\section{References}

1. Z. M. Zhou, Study on quality evaluation of Radix Rehmanniae\& processing method of Rehmanniae Radix Preparation [D], Henan University of Chinese Medicine, 2017.

2. F. F. Zhang, Effects of differently processed Rehmannia roots on Yin deficiency animals[D]. Shandong University, 2018.

3. M. Li, X. L. Wang, Z. G. Zhang, J. K. Zhang, X. Zhao, X. K. Zheng and W. S. Feng, Three new alkaloids and a new iridoid glycoside from the roots of Rehmannia glutinosa, Phytochem. Lett. 2017,21, 157-162.

4. R. X. Zhang, J. Zhou, M. X. Li, H. G. Ma, J. G. Qiu, X. H. Luo and Z. P. Jia, Ameliorating effect and potential mechanism of Rehmannia glutinosa oligosaccharides on the impaired glucose metabolism in chronic stress rats fed with high-fat diet, Phytomedicine. 2014, 21,607-614.

5. Z. Dong and C. X. Chen, Effect of catalpol on diabetic nephropathy in rats, Phytomedicine. 2013, 20, 1023-1029.

6. X. L. Zhang, C. Z. Jin, Y. C. Li, S. Guan, F. Han and S. P. Zhang, Catalpol improves cholinergic function and reduces inflammatory cytokines in the senescent mice induced by $\mathrm{D}$-galactose, Food Chemical Toxicology. 2013, 58, 50-55.

7. Y. H. Wang, M. Kwak, P. C. W. Lee and J. O. Jin, Rehmannia glutinosa polysaccharide promoted activation of human dendritic cells, International Journal of Biological Macromolecules. 2018, 116, 232-238.

8. Y. Huang, C. M. Jiang, Y. L. Hu, X. J. Zhao, C. Shi, Y. Yu, C. Liu, Y. Tao, H. R. Pan, Y. B. Feng, J. G. Liu, Y. Wu and D. Y. Wang, Immunoenhancement effect of rehmannia glutinosa polysaccharide on lymphocyte proliferation and dendritic cell, Carbohydrate Polymers. 2013, 96, 516-521. 
9. Q. Q. Han, Effects and analysis of different processing methods on the chemical compositions of the TCM Dihuang, Clinical Journal of Chinese Medicine. 2013, 5(9), 33-34.

10. X. Li, N. Wang, X. L. Wei and Z. L. Zhang, Changes in History and Modern Research of Radix Rehmanniae Charcoal and Radix Rehmanniae Praeparata Charcoal Processing, Chinese Archives of Traditional Chinese Medicine. 2012, 30(8), 1737-1739.

11. Y. Y. Kong, Z. L Zhang, W. N. Duan, Z.Q. Chen and M. L. Li, Comparative Study on Extraction of Effective Components of Catalpol in Rehmannia Glutinosa Libosch and Comparison of Catalpol Contents in Different Processed Products, Chinese Journal of Traditional Chinese Medicine, 2013,181(28):865-867

12. Z. J. Wang, G.D. Wei, and S. T. Ma, Chemical and Pharmacological Effects of Rehmanniae Radix Polysaccharides, Chinese Journal of Experimental Traditional Medical Formulae. 2015, 31(16), 231-234

13. H. M. Kim, C. S. An, K. Y. Jung, Y. K. Choo, J. K. Park and S. Y. Nam, Rehmannia glutinosa inhibits tumour necrosis factor-a and interleukin-1 secretion from mouse astrocytes, Pharmacological Research, 1999,40:171-176

14. Y. Cui, C. L. Rong, J. M. Wang, C. Cui, L. Wang, Z. Y. Feng, J. Feng and B. Niu, Mechanism-based antianxiety effects of polysaccharides extracted from Shudihuang (Radix Rehmanniae Preparata) by twodimensional electrophoresis analysis in rat hippocampus proteins, J. Tradition. Chin. Med. 2013, 33, 524-530.

15. H. W. Li and X. L. Meng, Research progress on chemical constituents and pharmacological activities of Rehmannia glutinosa,Drug Eval Res. 2015, 38,218-228.

16. X.J. Li, C. Jiang, N. Xu, J. X. Li, F. Y. Meng and H. Q. Zhai, Sorting and identification of Rehmannia glutinosa germplasm resources based on EST-SSR, scanning electron microscopy micromorphology, and quantitative taxonomy, Industrial Crops \& Products. 2018, 123, 303-314

17. Y. F. Liu, D. Liang, H. Luo, Z. Y. Hao, Y. Wang, C. L. Zhang, G. Ni, R. Y. Chen and D. Q. Yu, lonone glycosides from the roots of Rehmannia glutinosa, Journal.of Asian Natural Products Research. 2014, 16, 11-19.

18. Y. L. Zhang, W. S. Feng, X. K. Zheng, Y. G. Cao, Y. Y. Lv, H. Chen and H. X. Kuang, Three new ursane-type triterpenes from the leaves of Rehmannia glutinosa, Fitoterapia. 2013, 89, 15-19.

19. F. Q. Wang, J. Y. Zhi, Z. Y. Zhang, L. N. Wang, Y. F. Suo, C. X. Xie, M. J. Li, B. Zhang, J. F. Du, L. Gu and H. Z. Sun, Transcriptome analysis of salicylic acid treatment in Rehmannia glutinosa hairy roots using RNAseq technique for identification of genes involved in acteoside biosynthesis, Frontiers in Plant Science. $2017,8,1-15$.

20. F. Q. Wang, Y. F. Suo, H. Wei, M. J. Li, C. X. Xie, L. N. Wang, X. J. Chen and Z. Y. Zhang, Identification and characterization of 40 isolated Rehmannia glutinosa MYB family genes and their expression profiles in response to shading and continuous cropping, International Journal of Molecular Sciences, 2015, 16,15009-15030.

21. K. F. Fan, F. Y. Tang, J. P. Zhao, S. Q. Sun and Q. Zhou, Quantitative Analysis on the Processing End-point of Wine Stewed Rehmannia By FTIR, Chinese Journal of Veterinary Drug. 2017,51(9):49-54.

22. ]22] Z. J. Wang, G. D. Wei and S. T. Ma, Chemical Pharmacological Effects of Rehmannia Radix Polysaccharides. Chinese Journal of Experimental Medical Formulae. 2015, 21 (16) 231-34 
23. Z. Li, L.H. Zhou, Z. L. Ye and L. DING, Research progress on application of infrared reflectance spectroscopy in quality control of Chinese materia medica. Drug Evaluation Research, 2016, 39 (3): 465468

24. Z.Q.Sun, Q.M. Guo, Y. Ma and F.Q. Zhou, Research Advances of Thermal Analysis Application in Traditional Chinese Medicine. 2017, 19(1): 108-110

25. T.Mi and X-M Yu, Study on pyrolysis characteristics of medical waste, Journal of the Energy Institute 2012, 85 (3): 170-175

26. W.X, Fan, X.F. Lu, X.L Meng, Y.R. Lan, B.Z. Song, Q.L Mai and L.P. Chang, Thermal Decomposition Mechanism and Kinetic Parameters of Semen Ziziphi Spinosae Based on Thermogravimetric Analysis, Asian Journal of Chemistry, 2014, 26 (15): 4907-4911

27. M. I. Gao, B.H. Bao, Y D. Cao, M.Q. Shan, F.F Cheng, M Jiang, P.D. Chen * and L. Zhang, Article chemical property changes and thermal analysis during the carbonizing process of the pollen grains of typha, Molecules 2019, 24, 128:1-14.

28. Y.Q. Chen, H.B. He, C. Liu and X. H. Lu, Thermal Decomposition of Glucose and Sucrose by Kinetics Analysis, Chinese Journal of Process Engineering. 2010, 10 (4): 720-725

\section{Figures}
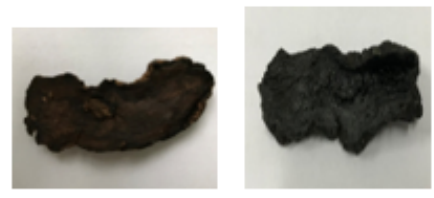

$\mathrm{RR}$

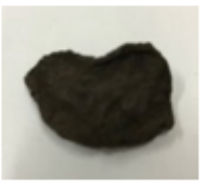

RRC

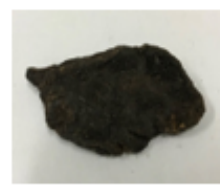

RRPC

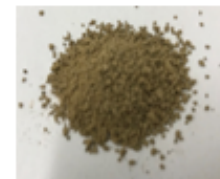

RRE

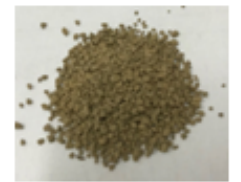

RRPE
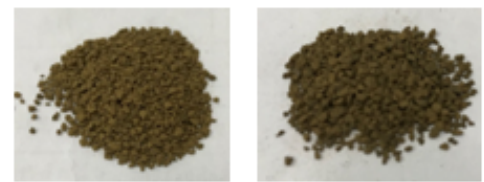

RRCE

RRPCE

Figure 1

Four types of R. glutinosa and their extracts 


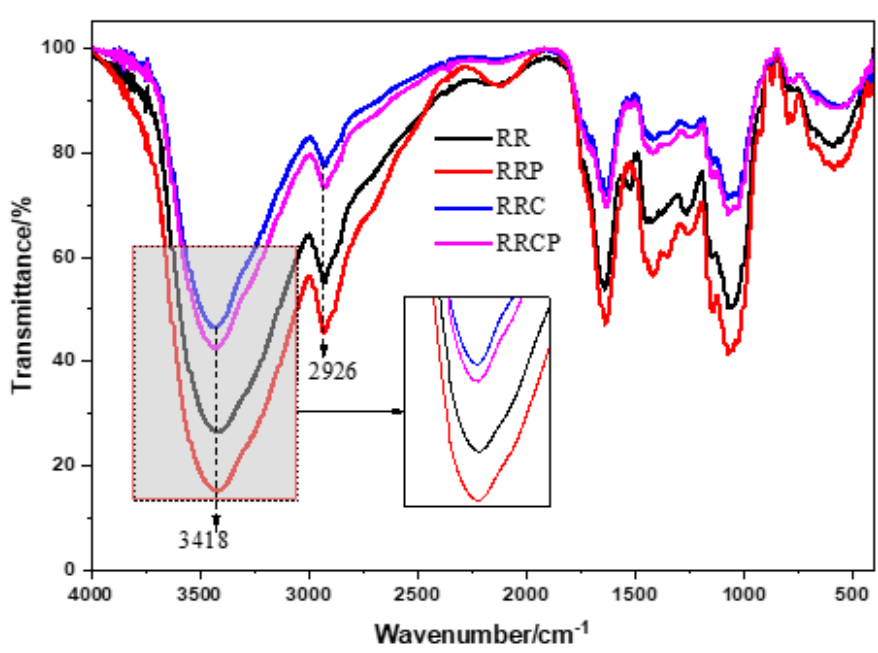

a RR, RRP, RRC, and RRCP

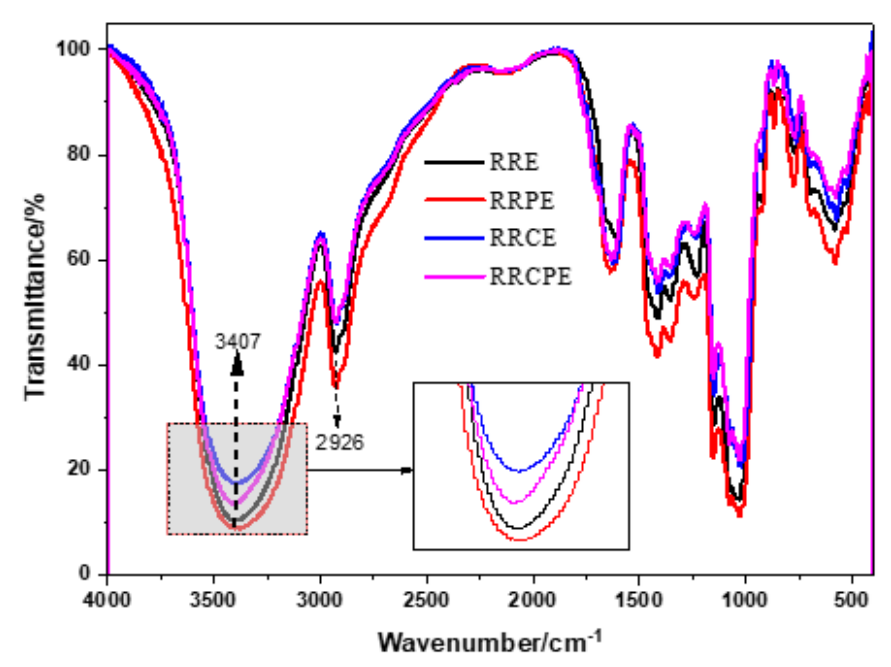

b RRE, RRPE, RRCE, and RRPCE

Figure 2

FTIR spectra of four types of $R$. glutinosa and their extracts 

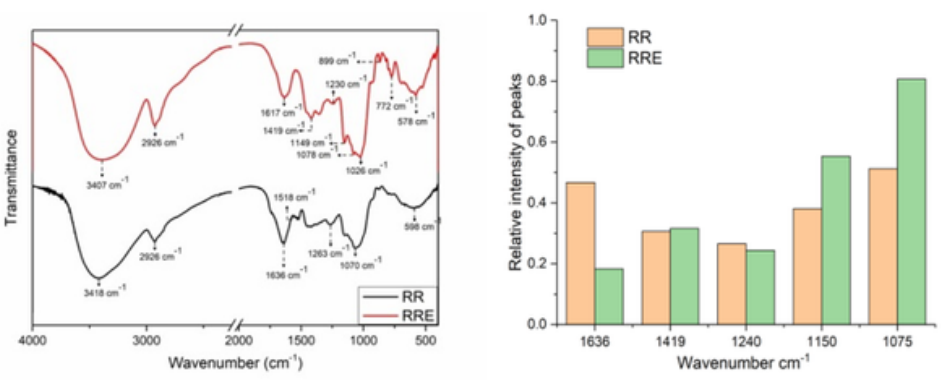

a RR and RRE
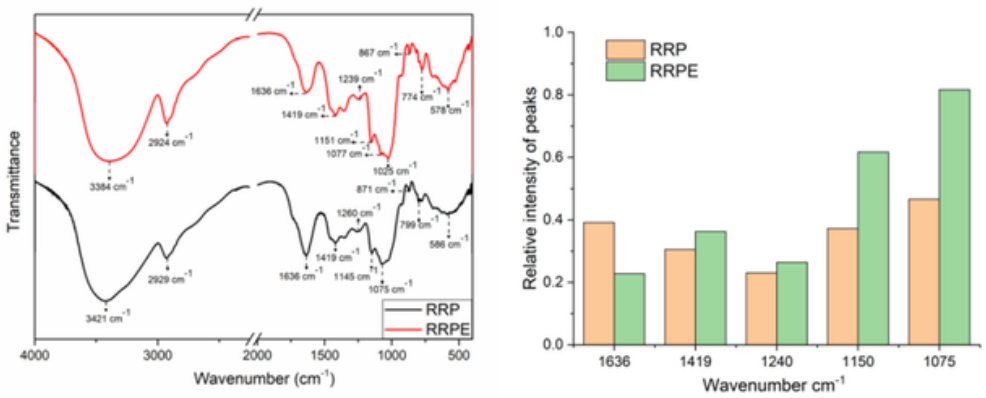

b RRP and RRPE
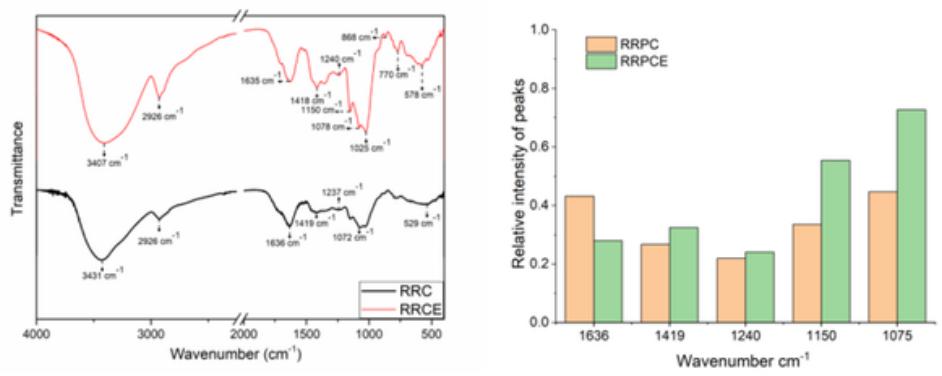

c RRC and RRCE
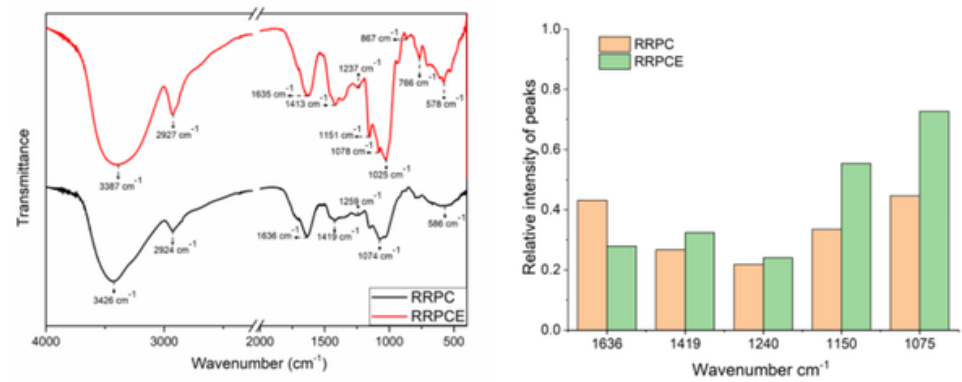

d RRCP and RRPCE

Figure 3

Comparison of FTIR spectra and the relative intensity of absorption peaks of extracts and their corresponding R. glutinosa 


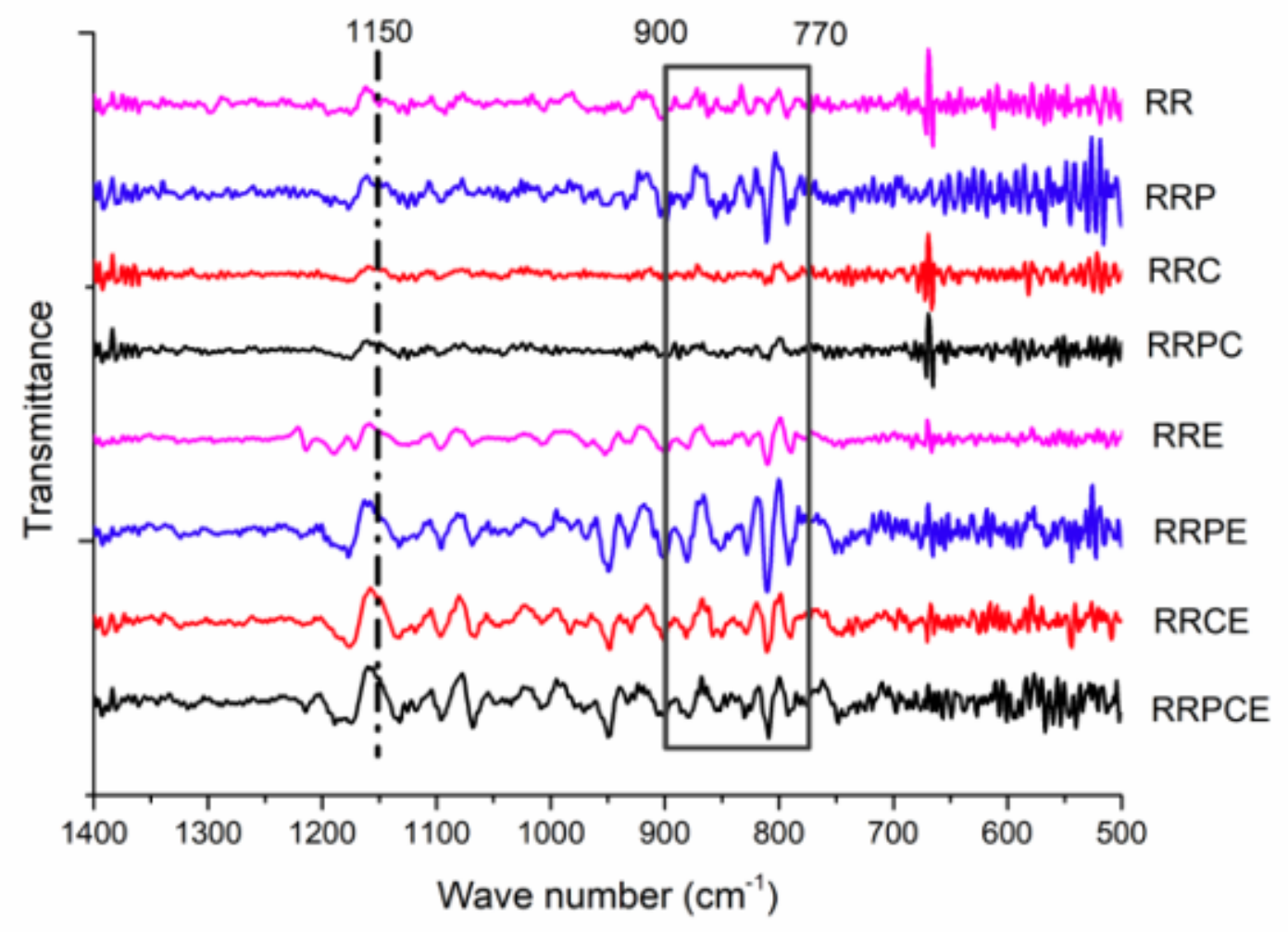

Figure 4

Second derivative spectra of four types of $R$. glutinosa and their extracts

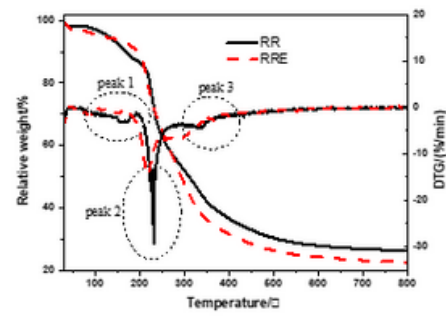

a RR and RRE

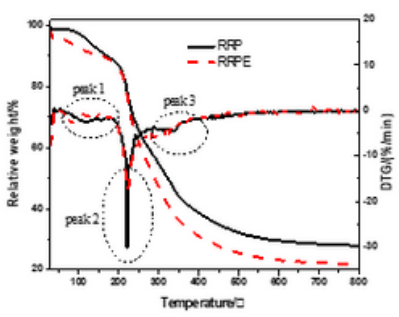

a RR and RRE

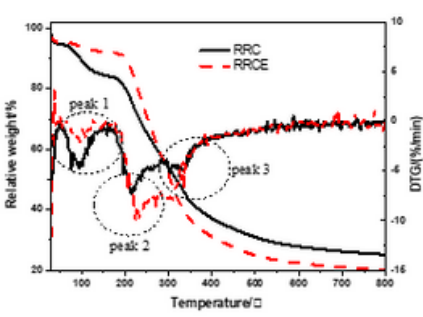

c RRC and RRCE

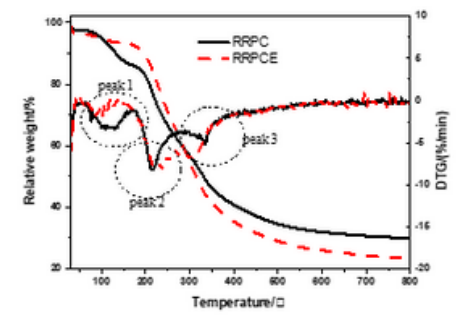

d RRPC and RRPCE

Figure 5

TG \& DSC curves of four types of $R$. glutinosa and their extracts. 